\title{
Knockdown long non-coding RNA PEG10 inhibits proliferation, migration and invasion of glioma cell line U251 by regulating miR-506
}

\author{
Junjun Liang ${ }^{1}$, Nina Liu ${ }^{2}$ and Haibin Xin ${ }^{1}$ \\ ${ }^{1}$ Department of Neurosurgery, Anqiu People's Hospital, Anqiu, China \\ ${ }^{2}$ Department of Neurology, Anqiu People's Hospital, Anqiu, China
}

\begin{abstract}
Glioma is a serious malignant tumor without effective therapies till now. IncRNA PEG10 was reported to have some biological activities in cancers. Hence, we explored the effects of PEG10 on the human glioma cell line U251 cells. U251 cells were transfected with sh-PEG10 and/or miR506 inhibitor. The expression of PEG10 and miR-506 was measured by qRT-PCR. Cell viability, cell apoptosis, cell migration and invasion were detected by CCK- 8 assay, flow cytometry and Transwell chamber assay, respectively. The cell proliferation and apoptosis related p16, p53, Bcl-2, Bax, and pro-/Cleaved-Caspase-3/9, migration and invasion related-protein: matrix metalloproteinases MMP-2, MMP-9 and vimentin, and Raf/MEK/ERK and JAK1/STAT3 pathways-related proteins were accessed by Western blot. Transfection with sh-PEG10 inhibited cell viability, migration and invasion, and increased cell apoptosis. Meanwhile, PEG10 silence upregulated the expression of p16 and p53, Bax, cleaved-Caspase-3/9 expression, and downregulated Bcl-2 expression. PEG10 silence upregulated miR-506 expression. Co-transfection with sh-PEG10 and miR-506 inhibitor impaired the tumor suppressive effects. PEG10 knockdown decreased the phosphorylation of Raf/MEK/ERK and JAK1/STAT3-related proteins Raf, MEK, ERK, JAK1 and STAT3. PEG10 knockdown inhibited cell viability, migration and invasion, induced cell apoptosis through miR-506 upregulation, as well as inactivation of Raf/MEK/ERK and JAK1/STAT3 signal pathways.
\end{abstract}

Key words: Glioma - PEG10 - miR-506 - Raf/MEK/ERK — JAK1/STAT3

\section{Introduction}

Glioma is a serious malignant tumor which belongs to one of the most common and fatal intracranial tumors ( $\mathrm{Li}$ et al. 2018b). This kind of cancer possesses features in widespread and fast invasion throughout the brain and what makes it worse due to its strongly resists to the traditional and newer targeted therapies (Winter et al. 2017). Even though more understanding about the pathogenesis is obtained, the outcome about survival percentage following surgery and prognostic factors of the patients is still not optimistic (Miller et al. 2017; Winter et al. 2017). New therapy or novel medicine was urgently needed for the diagnosis and treatment of gliomas.

Long non-coding RNAs (lncRNAs), which are longer than 200 nucleotides, do not have the functions of protein

Correspondence to: Haibin Xin, Department of Neurosurgery, Anqiu People's Hospital, No. 159 Xuefu Street, Anqiu 262100, China E-mail: xinhaibin0089@sina.com synthesis as the name described (Xing et al. 2014). Increasing evidence revealed that lncRNAs might be involved in various biological activities in several diseases, including glioma (Zhang et al. 2012; Necsulea et al. 2014). For example, lncRNA ADAM metallopeptidase with thrombospondin type 1 motif, 9 (ADAMTS9) antisense RNA 2 (ADAMTS9AS2) demonstrated anti-tumor functions in glioma via inhibiting cell migration (Yao et al. 2014); lncRNA taurine upregulated gene 1 (TUG1) could promote cell apoptosis to suppress glioma tumor ( $\mathrm{Li}$ et al. 2016b). Among all these experimental validated lncRNAs, paternally expressed 10 (PEG10, Gene ID: 23089) was first reported by Ono et al. and was a maternally imprinted and novel paternally expressed gene (Ono et al. 2001). Previous studies have suggested that PEG10 participated in modulating several biological processes in cancers, such as esophageal cancer (Tang et al. 2015), gastric cancer (Ishii et al. 2017) and hepatocellular carcinomas (Kobayashi 2014). However, from our limited knowledge, no studies were performed to explore the func- 
tions of PEG10 in glioma cells. Hence, we aimed to investigate the the roles of PEG10 in glioma.

miRNAs, which referred to a class of noncoding RNA species ( $22 \mathrm{nts})$ that are involved in gene regulation at the post-transcriptional levels (Chou et al. 2016). IncRNAs were also found to be interaction with miRNA, act as miRNA sponges (Paraskevopoulou et al. 2016), including PEG10. PEG10 was reported to be down-modulated by miR-122 to trigger its tumor suppressive effects in human hepatocellular carcinoma (Shyu et al. 2016). In addition, miR-491 and PEG10 could cooperate as an axis to influence cell proliferation and apoptosis in HCT-116 cells (Xu et al. 2013). miR-506 is a widely reported miRNA which functions as a tumor suppressor in glioma (Luo et al. 2015; Peng et al. 2016). Based on that, we further explored whether miR-506 and PEG10 could cooperate to function in glioma.

In this study, we used human glioma cell line U251 to establish cell model in vitro and investigated the effects of PEG10 on U251 cells as well as the underlying mechanisms. Our study might provide a new insight for the treatment of glioma.

\section{Material and Methods}

\section{Cell culture}

The human glioma cell line U251 was purchased from Procell life science and technology Co, Ltd (Cat. No: CL-0237, Wuhan, China). The specific growth medium for U251 cells were Dulbecco's modified Eagle medium (DMEM, Gibco, Grand Island, NY) with $10 \%$ fetal bovine serum (FBS, Gibco). Cells were cultured under the condition of $95 \%$ air with $5 \% \mathrm{CO}_{2}$ at the temperature of $37^{\circ} \mathrm{C}$. The culture medium was renewed every two to three days.

\section{Transfection}

In order to alter the expression of PEG10 and miR-506, sh-PEG10 (lncRNA PEG10 silence) and miR-506 inhibitor (miR-506 silence) and their corresponding negative control (NC) (GenePharma Co, Shanghai, China) were transfected into U251 cells. Pre-treated cells at the density of $2 \times 10^{5}$ cells/well were seeded and incubated until the cells arrived at 70-80\% confluence, they were transfected with sh-PEG10, miR-506 inhibitor and NC using Lipofectamine 2000 reagent (Invitrogen).

\section{Quantitative real-time polymerase chain reaction ( $q R T-P C R$ )}

Total RNA was obtained from U251 cells using Trizol reagent (Invitrogen, Carlsbad, CA, USA). The Taqman MicroRNA Reverse Transcription Kit (Cat. No: 4366596, Thermo Fisher Scientific, Rockford, IL, USA) was used for converting miRNA to cDNA. The Taqman Universal Master Mix II
(Cat. No: 4440040, Thermo Fisher Scientific) was used for cDNA be generated in a reverse transcription reaction. These two cooperate with TaqMan MicroRNA Assay of PEG10 and miR-506 (Thermo Fisher Scientific) were used for determining the PEG10 and miR-506 expression in U251 cells. U6 and GAPDH were used for the internal controls for miR-506 and PEG10, respectively.

\section{Cell viability assay}

Cell viability was enacted by cell Counting Kit-8 (CCK-8, Yeasen, Shanghai, China). Firstly, U251 cells were seeded in in 96 -well plate. Cells were maintained at $37^{\circ} \mathrm{C}$ in humidified air with $5 \% \mathrm{CO}_{2}$. Secondly, added $10 \mu \mathrm{CCK}-8$ solution and cells were incubated for $1 \mathrm{~h}$. After incubation, absorption values for detecting cell viability were read at $450 \mathrm{~nm}$ using a Microplate Reader (Bio-Rad, Hercules, CA).

\section{Apoptosis assay}

Propidium iodide (PI) and fluorescein isothiocyanate (FITC)-conjugated Annexin V staining (Yeasen, Shanghai, China) was used for determining cell apoptosis. In brief, the cells at the density of 100,000 cells/well were seeded in 6 well-plate. Treated cells were washed twice with precooling phosphate buffer saline (PBS) and centrifuged to resuspend in binding buffer. Then added $5 \mu$ Annexin V-FITC, mixed gently and put in the dark and incubated for $15 \mathrm{~min}$. In addition, added $5 \mu \mathrm{PI}$ to the plates. The apoptotic cells rate was measured with flow cytometer (Beckman Coulter, USA) according to the manufacture's instruction.

\section{Migration and invasion assay}

Cell migration was evaluated by using a modified twochamber migration assay (pore size: $8 \mu \mathrm{m}$ ). Cell suspension $100 \mu \mathrm{l}$ (around $2 \times 10^{5}$ cells $/ \mathrm{ml}$ ) without serum was added to upper Transwell. Then $600 \mu$ culture medium with $10 \%$ FBS was added in the lower Transwell. U251 cells were maintained for $24 \mathrm{~h}$ at $37^{\circ} \mathrm{C}$ with $95 \%$ air and $5 \% \mathrm{CO}_{2}$. After incubation, cells at the upper surface of the filter were removed by a cotton swab, and the filter was fixed with methanol for $5 \mathrm{~min}$. U251 cells at the lower surface of the filter were stained by crystal violet for $15 \mathrm{~min}$. Cells were counted under microscope (100× magnification). Cell invasion was conducted in the similar process as cell migration, but the inserts were coated with $50 \mu \mathrm{g}$ of Matrigel (BD Biosciences, Bedford, MA, USA).

\section{Western blot}

Western blot was used in our study to detect all the proteins expression. Proteins were obtained from U251 cells using 
RIPA lysis buffer (Cat. No: R0010, Solarbio, Beijing, China) supplemented with protease inhibitors (Thermo Fisher Scientific). The $\mathrm{BCA}^{\mathrm{Tm}}$ Protein Assay Kit (Pierce, Appleton, WI, USA) was used for determining proteins concentration. The Western blot system was established using a Bio-Rad BisTris Gel system following the manufacturer's instructions. Primary antibodies included: anti-p16 antibody (ab51243), anti-p53 antibody (ab75754), anti-B-cell lymphoma 2 (Bcl-2) antibody (ab32124), anti-Bcl-2-associated X protein (Bax) antibody (ab32503), anti-pro Caspase-3 antibody (ab32499), anti-pro Caspase-9 antibody (ab135544), anti-cleaved Caspase- 3 antibody (ab32042), anti-cleaved Caspase- 9 antibody (ab2324), anti-matrix metalloproteinases MMP-2 antibody (ab92536), anti-MMP-9 antibody (ab76003), anti-vimentin antibody (ab137321), , anti-beta actin antibody (ab8227), all from Abcam (Cambridge, UK); anti-Raf antibody (53745), anti-extracellular signal-regulated kinase (ERK) antibody (4695), anti-phospho-ERK, antibody (4370), anti-mitogenactivated protein kinase ERK kinase (MEK) antibody (9126), anti-phospho-MEK, antibody (9154), anti-janus kinase (JAK) 1 antibody (3344), anti-phospho-JAK1 antibody (74129), anti-signal transducers and activator of transcription (STAT) 3 antibody (12640), anti-phospho-STAT3 antibody (9145), all from Cell Signaling Technology (Beverly, MA, USA). Primary antibodies were prepared in 5\% blocking buffer and diluted according to the product instruction. These primary antibodies were incubated in membrane and maintained at $4^{\circ} \mathrm{C}$ overnight at recommended concentration. Then for second antibody incubation, incubate with horseradish peroxidase (HRP) conjugated second antibody. Detection was performed by capturing the signals and analyzing the intensity of the bands was quantified using Image $\mathrm{Lab}^{\mathrm{TM}}$ Software (Bio-Rad, Shanghai, China).

\section{Statistical analysis}

Results are shown as the mean \pm standard deviation (SD) of three independent experiments. Statistical analyses were performed using GraphPad 6.0 statistical software (GraphPad, San Diego, CA, USA). The $p$-values were calculated using a one-way analysis of variance (ANOVA). $p<0.05, p<$ 0.01 , and $p<0.001$ reveal statistically significant differences.

\section{Results}

PEG10 knockdown inhibited cell proliferation and induced cell apoptosis

To clarify the functions of PEG10, U251 cells were transfected with sh-PEG10 to silence PEG10. The expression of PEG10 was significantly downregulated, indicating high transfection efficiency ( $p<0.01$, Figure 1A). Cell viability, cell apoptosis and their corresponding factors were detected. Results showed that transfection with sh-PEG10 significantly decreased cell viability within each time interval especially in $96 \mathrm{~h}(p<$ 0.01 , Figure $1 \mathrm{~B}$ ). Based on this, we verified the expression of proliferation-related factors p16 and p53. Obviously, we found that the expression of $\mathrm{p} 16$ and $\mathrm{p} 53$ was upregulated by transfection with sh-PEG10 (both $p<0.05$, Figures 1 C and D). In addition, we found that cell apoptosis was significantly increased by transfection with PEG10 in U251 cells $(p<0.01$, Figure 1E). Moreover, the expression of anti-apoptotic protein $\mathrm{Bcl}-2$ was downregulated while pro-apoptotic proteins Bax, cleaved-Caspase- 3 and cleaved-Caspase- 9 were upregulated by transfection with sh-PEG10 (Figure 1F). Taken together, transfection with sh-PEG10 in U251 cell inhibited cell growth by decreasing cell viability and increased cell apoptosis.

\section{PEG10 knockdown inhibited cell migration and invasion}

Cell migration and invasion are vital for cancer cells moving forward to the target tissue (Hood and Cheresh 2002). Hence, we measured the effects of sh-PEG10 transfection in U251 cells. Results showed that transfection with sh-PEG10 significantly decreased cell migration and cell invasion (both $p<0.05$, Figures $2 \mathrm{~A}$ and D). Consistent with this, we found that the expression of MMP-2, MMP-9 (Figures 2B and C) and vimentin (Figures $2 \mathrm{E}$ and $\mathrm{F}$ ) were all downregulated by transfection with PEG10 (all $p<0.05$ ). In a word, transfection with sh-PEG10 inhibited cell migration and invasion in U251 cells.

\section{PEG10 knockdown upregulated the expression of miR-506}

MiR-506 has been revealed to have tumor suppressive functions in glioma (Peng et al. 2016). Herewith, we proposed that miR-506 might also associate with the anti-tumor effects of sh-PEG10 in glioma cell line U251 cells. Results in Figure 3 demonstrated that transfection with sh-PEG10 significantly upregulated the expression of miR-506 compared with NC $(p<0.05)$, which indicated that miR-506 might be involved in the anti-tumor effects of PEG10 downregulation.

\section{PEG10 knockdown decreased cell viability and induced cell apoptosis via miR-506 upregulation}

To further explore the functions of miR-506, miR-506 inhibitor and NC were transfected into U251 cells. Statistically downregulation of miR-506 by transfection with miR-506 inhibitor indicated high transfection efficiency $(p<0.01$, Figure 4A). We experimentally determined cell viability, cell apoptosis and the related factors. Surprisingly, co-transfection with sh-PEG10 and miR-506 inhibitor impaired the anti-tumor effects of transfection with sh-PEG10 by increasing cell viability $(p<0.05$, Figure $4 \mathrm{~B})$ and increasing cell 
apoptosis ( $p<0.05$, Figure $4 \mathrm{E})$. In addition, the expression of corresponding factors by co-transfection with sh-PEG10 and miR-506 inhibitor presented in Figures 4C, D and F led to the opposite results with transfection with sh-PEG10 in U251 cells. Taken together, the anti-tumor effects of PEG10 knockdown were disappeared after transfected miR-506 inhibitor into cells, which indicated that the PEG10 knockdown decreased cell viability and induced cell apoptosis via miR-506 upregulation.
PEG10 knockdown decreased cell migration and invasion via miR-506 upregulation

How does transfection with miR-506 influence the effects of PEG10 knockdown? Similarly we evaluated the effects of co-transfection with sh-PEG10 and miR-506 inhibitor on $\mathrm{U} 251$ cells. We found that both migration and invasion were induced after transfection with miR-506 (both $p<0.05$, Figures 5A and D). Furthermore, the expression of MMP-2,
A
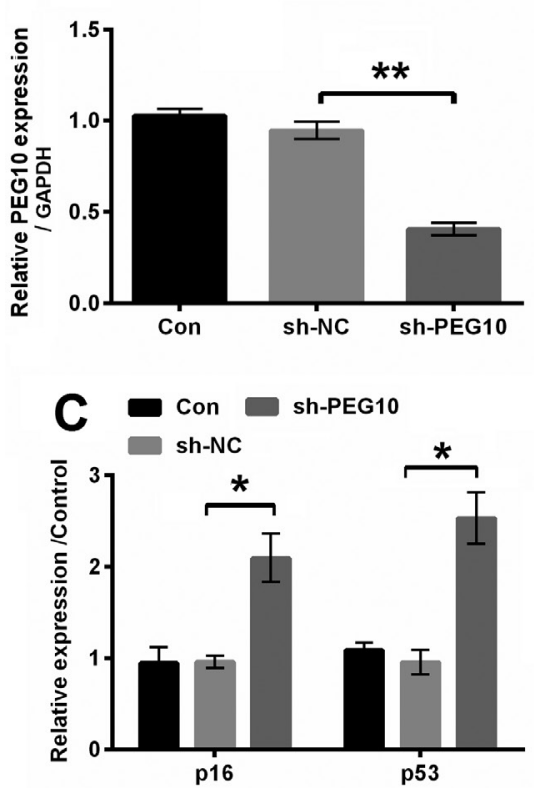

$\mathbf{E}$

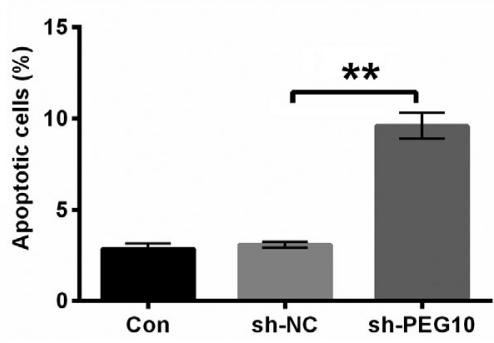

B

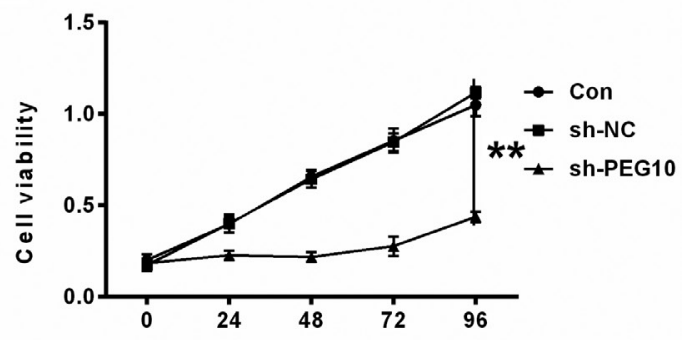

(h)

D

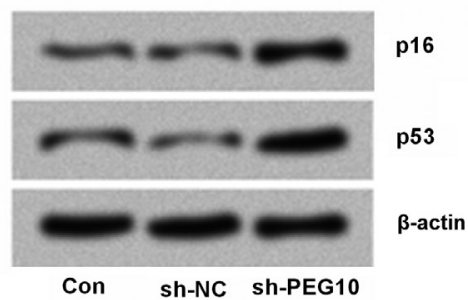

$\mathbf{F}$

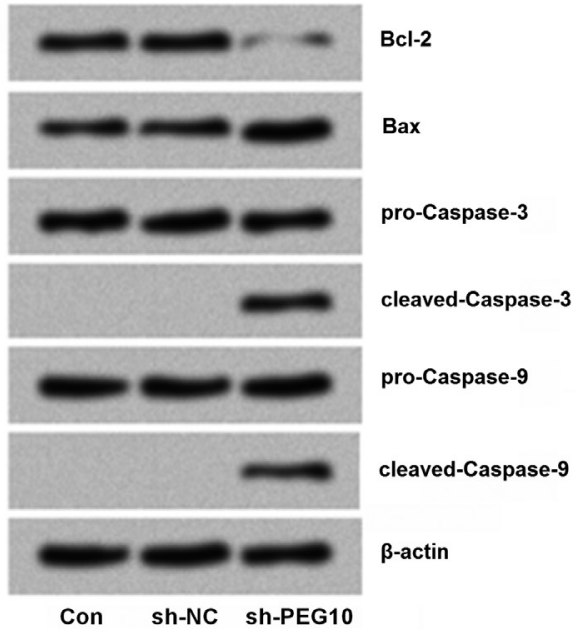

Figure 1. PEG10 downregulation inhibited cell viability and induced cell apoptosis in U251 cells. sh-PEG10 was transfected into U251 cells (A). Transfection with sh-PEG10 decreased cell viability in a time-dependent manner $(0,24,48,72$ and 96 h; B), upregulated the expression of p16 and p53 (C, D), increased cell apoptosis (E), and upregulated the expression of Bax, cleaved Caspase-3 and cleaved Caspase-9, and downregulated the expression of Bcl-2 (F) compared with transfection with sh-NC group. The data are shown as the mean \pm SD. ${ }^{*} p<0.05$ and $\left.{ }^{* *} p<0.01\right)$ are both considered as statistically significant. 
MMP-9 and vimentin were all upregulated (all $p<0.05$, Figures 5B, C and F). Overall, these results suggested that PEG10 knockdown decreased cell migration and invasion via modulating miR-506 expression.

\section{PEG10 knockdown inactivated Raf/MEK/ERK and JAK1/ STAT3 signal pathways by upregulation of miR-506}

The expression of Raf, the phosphorylation of MEK and ERK were downregulated by transfection with sh-PEG10 (all $p<0.05$ ) while were reversed by co-transfection with sh-PEG10 and miR-506 inhibitor (all $p<0.01$, Figures 6A and B). Similarly, the phosphorylation of JAK1 and STAT3 were downregulated by PEG10 knockdown while upregulated by transfection with sh-PEG10 and miR-506 inhibitor $(p<0.01$, Figures 6C and D). Taken together, treatment with
PEG10 knockdown inactivated Raf/MEK/ERK and JAK1/ STAT3 signal pathways by upregulation of miR-506.

\section{Discussion}

In the present study, we investigated the effects of lncRNA PEG10 on human glioma cell line U251 cells in vitro. The main results showed that PEG10 downregulation presented tumor suppressive effects by decreasing cell viability, inducing cell apoptosis, alleviating cell migration and invasion. Further experiment showed that PEG10 knockdown upregulated the expression of miR-506. In addition, the tumor suppressive effects of PEG10 knockdown were through upregulation of miR-506. This process might be modulated by inactivating of Raf/MEK/ERK and JAK1/STAT3 signal pathways.
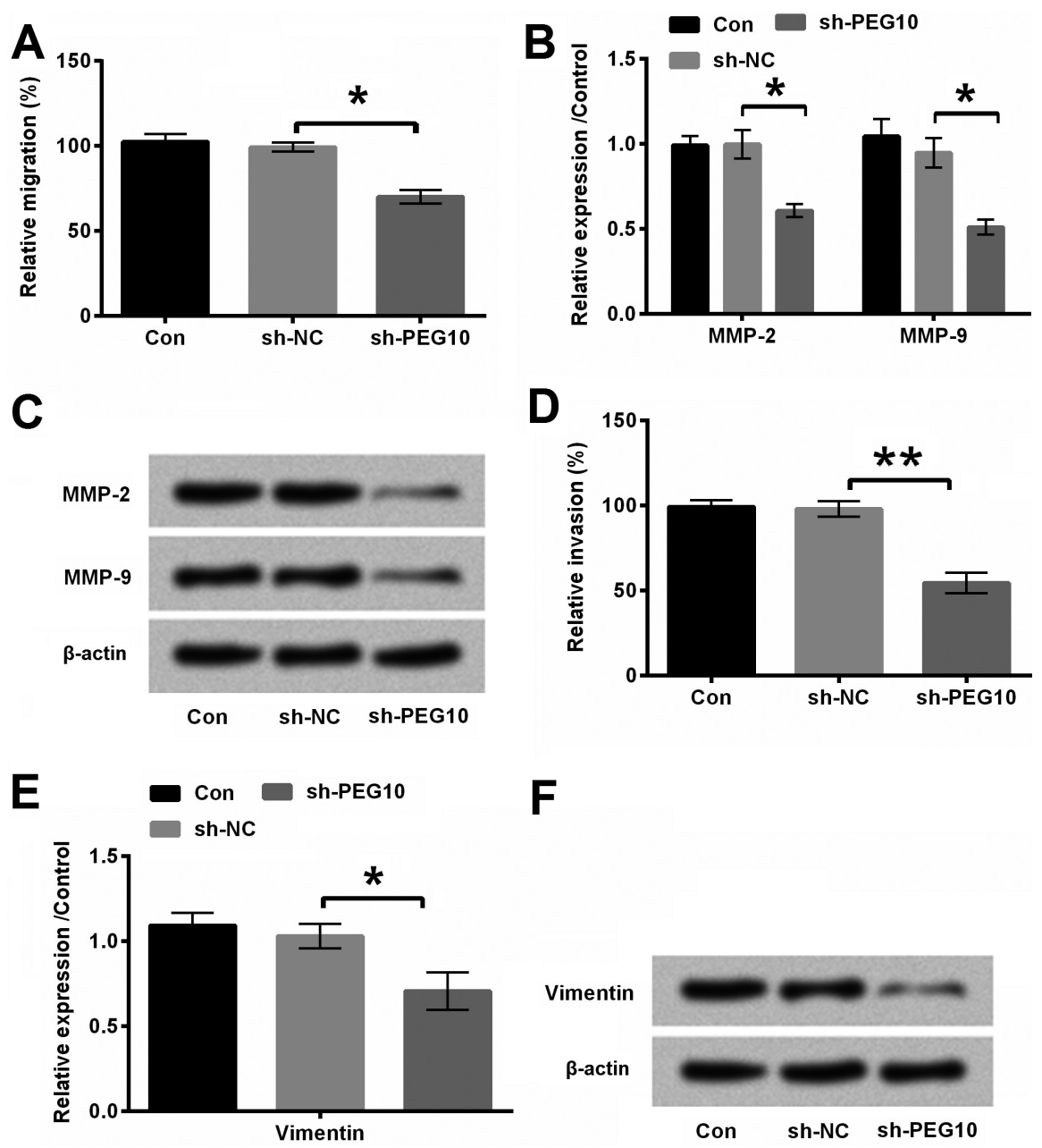

Figure 2. PEG10 downregulation inhibited cell migration and invasion. Transfection with sh-PEG10 decreased cell migration (A), downregulated the expression of matrix metalloproteinases MMP-2 and MMP-9 (B, C), inhibited cell invasion (D), downregulated the expression of vimentin (E, F) compared with transfection with sh-NC. The data are shown as the mean \pm SD. ${ }^{\star} p<0.05$ and ${ }^{* *} p<0.01$ are both considered as statistically significant. 


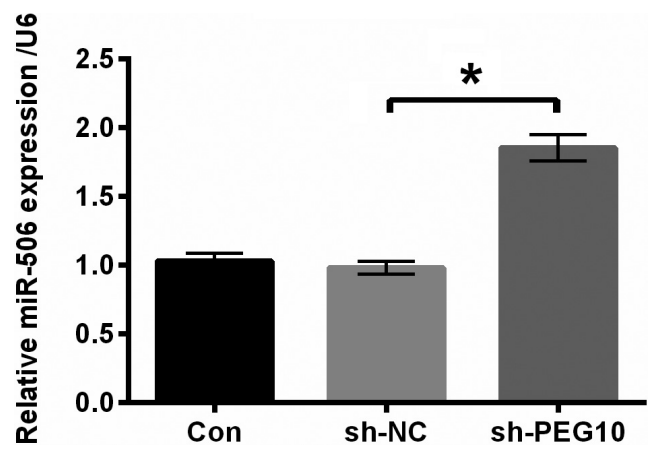

Figure 3. PEG10 downregulation upregulated the expression of miR-506. The data are shown as the mean $\pm \mathrm{SD}$. ${ }^{\star} p<0.05$ is considered as statistically significant.
Malignant glioma is the most catastrophic disease and often led to widespread and progressive disability and death. What make it worse is its difficult to diagnose and no standard effective treatments till now (Stewart 2002). lncRNAs demonstrated potential effects on glioma cell biogenesis, development and differentiation of gliomas (Zhang et al. 2012). In our study, we investigated the effects of lncRNA PEG10 expression on glioma cell growth. Results revealed that PEG10 knockdown inhibited cell growth by increasing cell viability and induced cell apoptosis. Previous studies from Hiroshi Okabe et al. found that PEG10 upregulation decreased cell apoptosis in human hepatocellular carcinogenesis, which supported our results on the other side (Okabe et al. 2003).

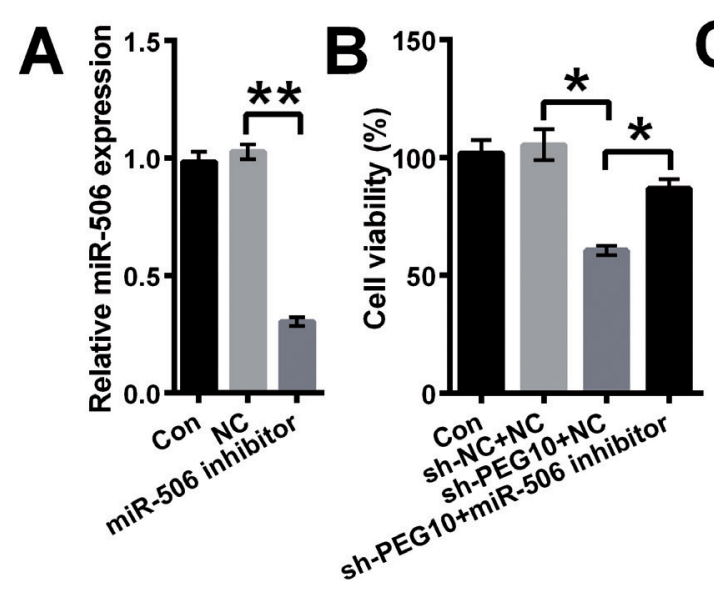

E

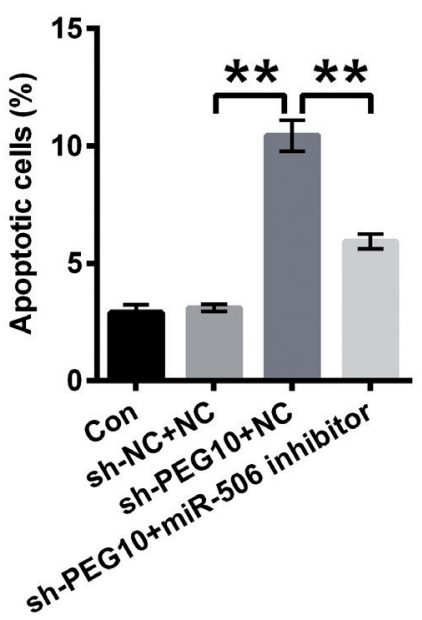

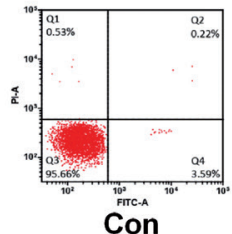
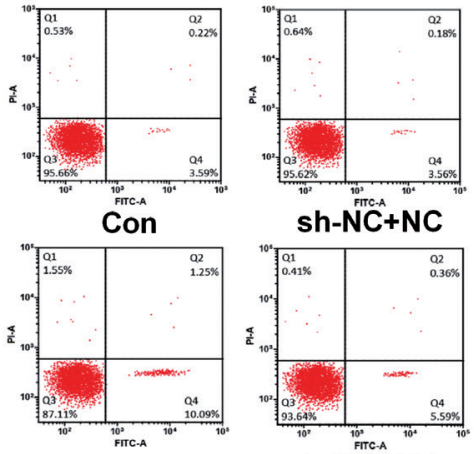

sh-PEG10+NC

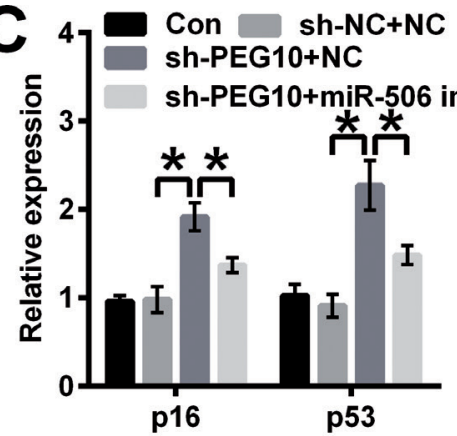

$\mathbf{F}$

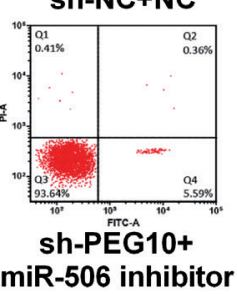

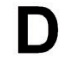

bitor

p16

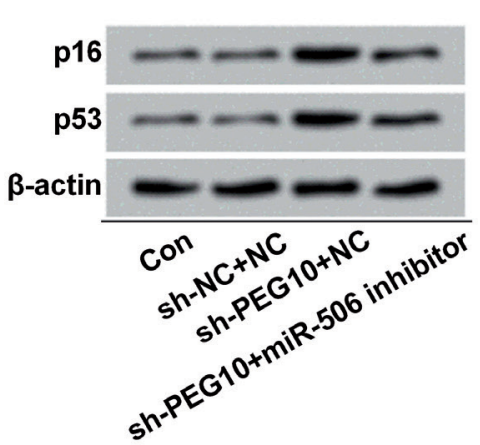

$\mathrm{Bcl}-2$

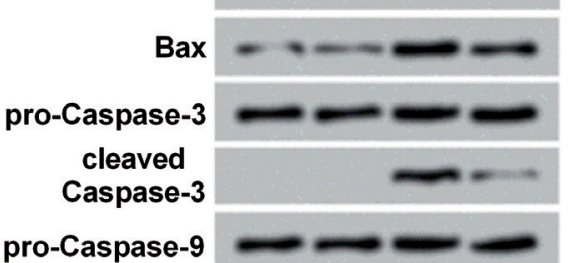

pro-Caspase-9

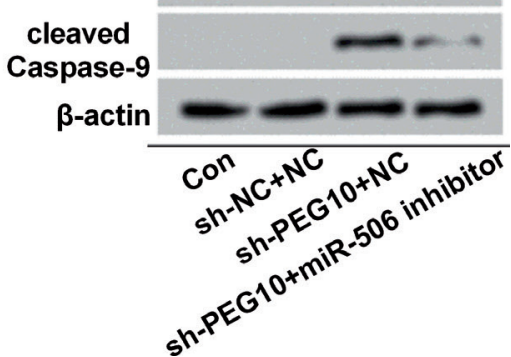

Figure 4. PEG10 downregulation decreased cell viability and induced cell apoptosis by miR-506 upregulation miR-506 inhibitor transfected into U251 cells (A). Co-transfection with sh-PEG10 and miR-506 inhibitor impaired the tumor suppressive effects by increasing cell viability (B), downregulating expression of p16 and p53 (C, D), decreasing cell apoptosis (E), upregulating Bcl-2 and downregulating Bax, cleaved-Caspase-3 and cleaved-Caspase-9 (F) compared with transfection with sh-PEG10 and NC. The data are shown as the mean $\pm \mathrm{SD} .{ }^{*} p<0.05$ and ${ }^{* *} p<0.01$ are both considered as statistically significant. 
In addition, migration and invasion are features of malignant cancer and cancer therapeutics are aimed to slow down tumor progress in migration and invasion (Friedl and Wolf 2003). Importantly, glioma was characterized by its widespread and rapid migration and invasion which made it difficult to treat (Miller et al. 2017). In our study, gladly, we found that PEG10 knockdown dramatically reduced cell migration and invasion, indicating the potential tumor suppressive effects of PEG10 knockdown. Our results supported these previous studies that PEG10 overexpression enhanced cell migration and invasion in human breast cancer (Li et al. 2016c), pancreatic cancer (Peng et al. 2017) and esophageal cancer cells (Zang et al. 2015).

We further explored the underlying mechanisms about how PEG10 knockdown could trigger tumor suppressive effects in U251 cells. Previous study pointed out that the functions of lncRNAs are often found to be connected with miRNAs which are involved in most of biological activities (Jalali et al. 2013). Therefore, we hypothesized that lncRNA PEG10 achieved its functions through regulating miRNAs. As mentioned in the forgone literature, lncRNA PEG10 affected gastric cancer cell growth and development through
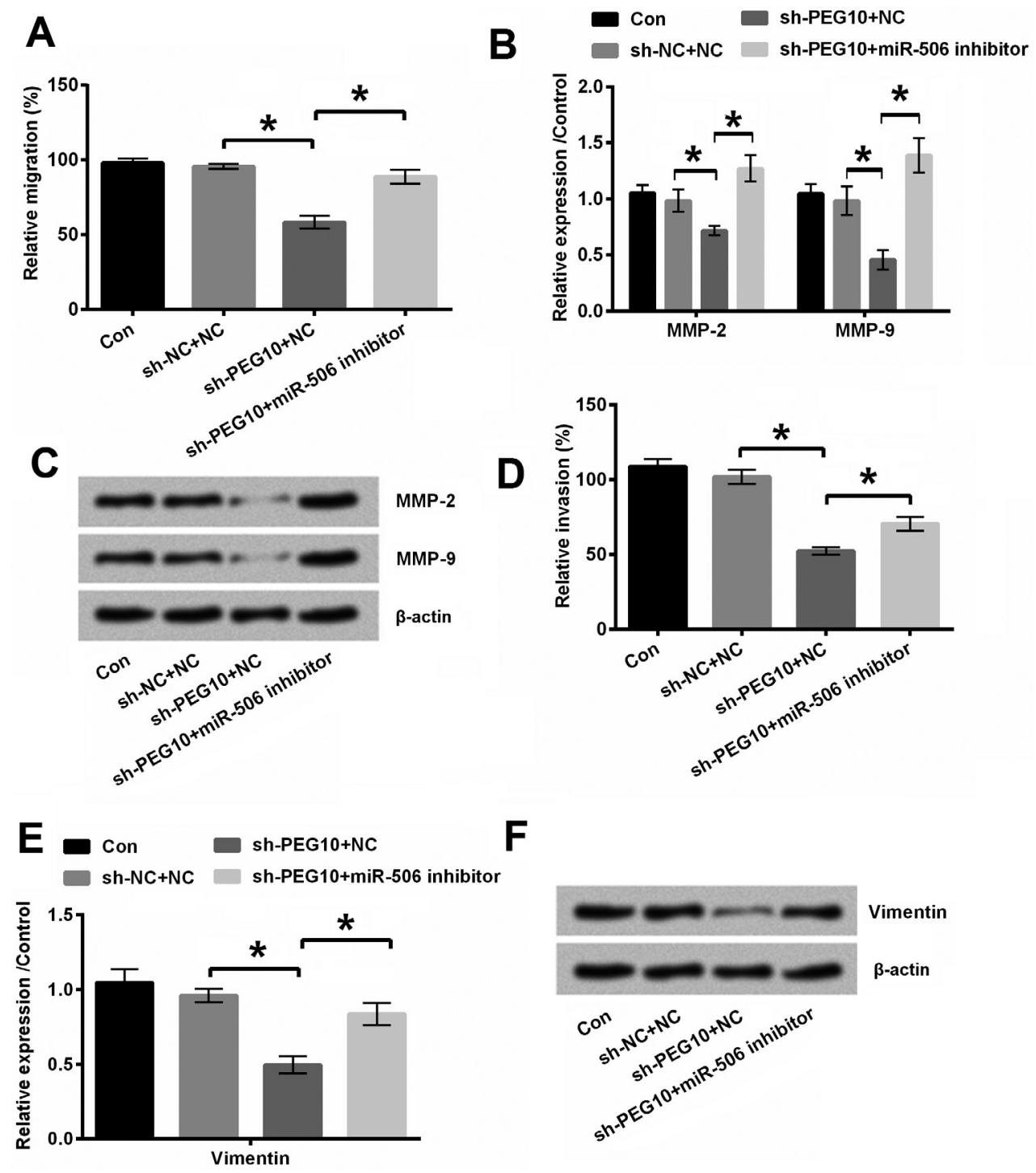

$\mathbf{F}$

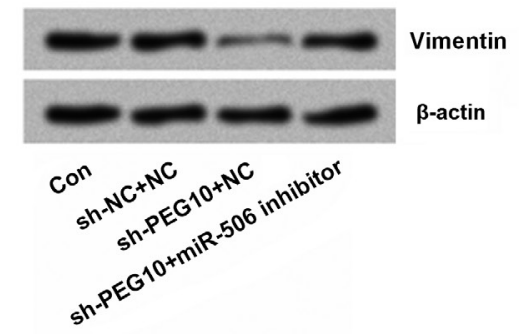

Figure 5. PEG10 downregulation decreased cell migration and invasion by miR-506 upregulation. Co-transfection with sh-PEG10 and miR-506 inhibitor increased cell migration (A) upregulated the expression of matrix metalloproteinases MMP-2 and MMP-9 (B, C), increased cell invasion (D), upregulated the expression of vimentin (E, F) compared with transfection with sh-PEG10 and NC. The data are shown as the mean \pm SD. ${ }^{\star} p<0.05$ is considered as statistically significant. 
regulation of miR-3200 (Wang et al. 2018), and miR-491/ PEG10 axis functions in modulating HCT-116 cells growth (Li et al. 2018a). Even though several recent studies started to pay attention to explore miRNAs and lncRNA PEG10, there are still more possibilities in this field. In our study, we induced miR-506 to investigate the possibility between PEG10 and miR-506. miR-506 was a well-studied tumor suppressor in various cancers, such as nasopharyngeal carcinoma (Zhang et al. 2015), colorectal cancer (Chen et al. 2015), pancreatic cancer (Li et al. 2016a) and in glioma (Luo et al. 2015; Peng et al. 2016). In our study, it is the first time that the correlation between lncRNA PEG10 and miR-506 was studied. Surprisingly, after transfection with miR-506 inhibitor into U251 cells, the anti-tumor effects of PEG10 knockdown was impaired with increased cell viability, migration and invasion, and inhibited cell apoptosis. In agreement with our work, we found that the roles of miR-506 in glioma
U251 cells were consistent with what roles miR-506 possess in the other cancers (Chen et al. 2015; Luo et al. 2015; Zhang et al. 2015; Li et al. 2016a; Peng et al. 2016) that miR-506 have anticarcinogenic functions. On the other hand, our study also revealed that the mechanism about how PEG10 knockdown could inhibit glioma cell growth and metastasis, that is by miR-506 upregulation.

Raf/MEK/ERK and JAK1/STAT3 pathways play vital roles in tumorigenesis on cell growth, apoptosis, cell cycle arrest and inducible resistance to drugs (McCubrey et al. 2007; Wen et al. 2014). In addition, Raf/MEK/ERK and JAK1/STAT3 pathway were also reported to be correlated in glioma (Liu et al. 2014; Malla et al. 2011). Inactivation of both of these signal pathways can be treated as resultant anti-tumor effect (De Luca et al. 2012; van der Zee et al. 2015). In our study, transfection with PEG10 inactivated both of Raf/MEK/ERK and JAK1/STAT3 pathways while transfection with miR-
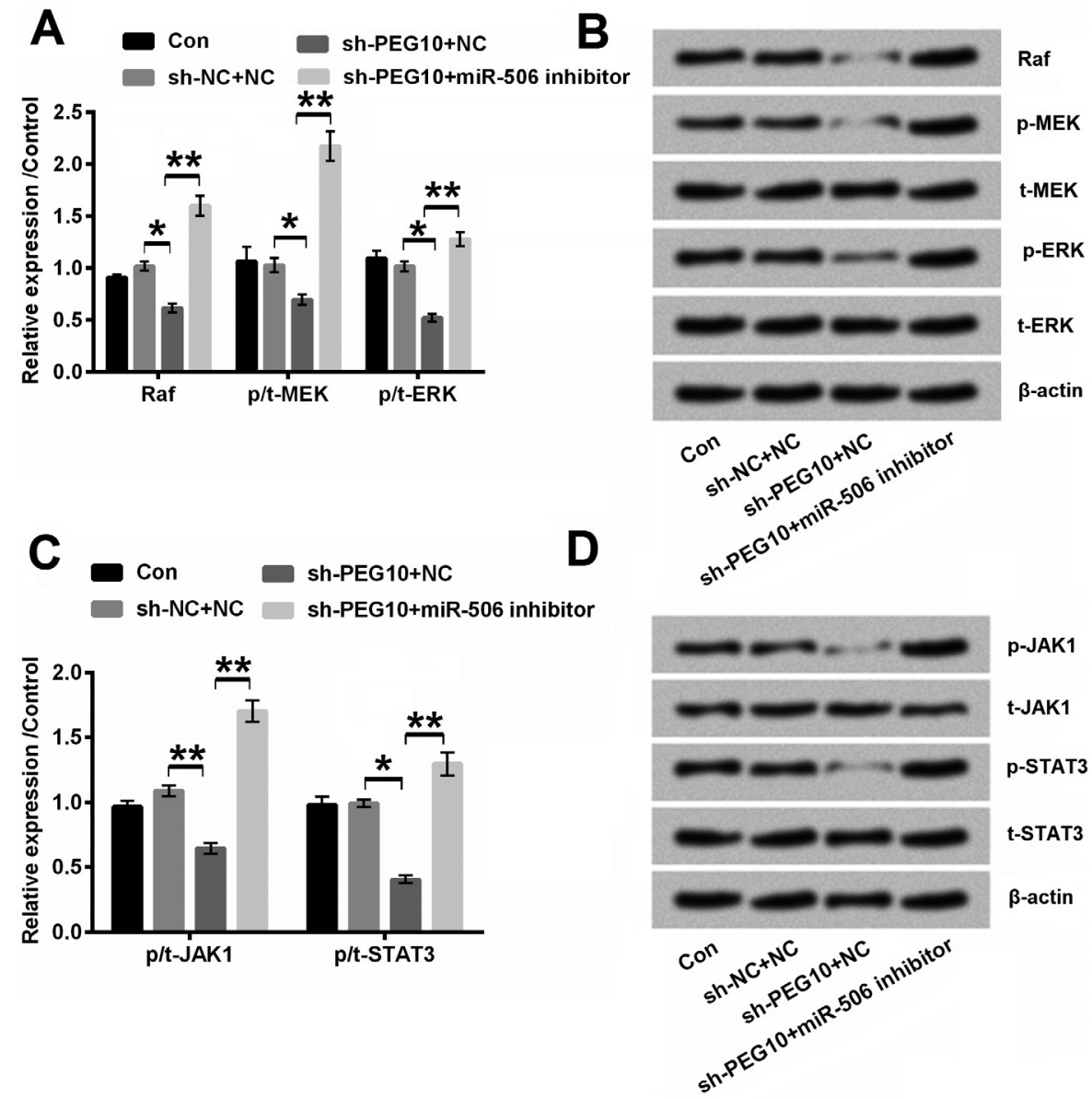

Figure 6. PEG10 downregulation inactivated Raf/MEK/ERK and JAK1/STAT3 signal pathways. Transfection with sh-PEG10 downregulated the expression of Raf, decreased the ratio of p/t-MEK and p/t-ERK (A, B), decreased the ratio of p/t-JAK1 and p/t-STAT3 (C, D) compared with control. Of contrast, co-transfection with sh-PEG10 and miR-506 inhibitor reversed this trend. The data are shown as the mean \pm SD. ${ }^{*} p<0.05$ and ${ }^{* *} p<0.01$ are both considered as statistically significant. ERK, extracellular signal-regulated kinase; MEK, mitogen-activated protein kinase ERK kinase; JAK, janus kinase; STAT, signal transducers and activator of transcription. 
506 inhibitor reversed these results which indicated that the effects of PEG10 knockdown in U251 cells could be via modulating of signal pathways by upregulation of miR-506.

In conclusion, our study revealed that PEG10 knockdown inhibited glioma cell growth and migration and invasion through regulation of miR-506. This process might be through inactivation of Raf/MEK/ERK and JAK1/STAT3 pathways. Further studies need to be done whether PEG10 could be treated as a biomarker for the treatment of glioma in the further.

Funding. This research received no specific grant from any funding agency in the public, commercial or not-for-profit sectors.

Conflict of interest. The authors declare no conflict of interest.

\section{References}

Chen Z, Liu S, Tian L, Wu M, Ai F, Tang W, Zhao L, Ding J, Zhang L, Tang A (2015): miR-124 and miR-506 inhibit colorectal cancer progression by targeting DNMT3B and DNMT1. Oncotarget 6, 38139-38150 https://doi.org/10.18632/oncotarget.5709

Chou CH, Chang NW, Shrestha S, Hsu SD, Lin YL, Lee WH, Yang CD, Hong HC, Wei TY, Tu SJ, et al. (2016): miRTarBase 2016: updates to the experimentally validated miRNA-target interactions database. Nucleic Acids Res. 44, D239-247 https://doi.org/10.1093/nar/gkv1258

De Luca A, Maiello MR, D'Alessio A, Pergameno M, Normanno N (2012): The RAS/RAF/MEK/ERK and the PI3K/AKT signalling pathways: role in cancer pathogenesis and implications for therapeutic approaches. Expert Opin. Ther. Targets 16 (Suppl. 2), S17-27

https://doi.org/10.1517/14728222.2011.639361

Friedl P, Wolf K (2003): Tumour-cell invasion and migration: diversity and escape mechanisms. Nat. Rev. Cancer 3, 362-374 https://doi.org/10.1038/nrc1075

Hood JD, Cheresh DA (2002): Role of integrins in cell invasion and migration. Nat. Rev. Cancer 2, 91-100 https://doi.org/10.1038/nrc727

Ishii S, Yamashita K, Harada H, Ushiku H, Tanaka T, Nishizawa N, Yokoi K, Washio M, Ema A, Mieno H, et al. (2017): The H19-PEG10/IGF2BP3 axis promotes gastric cancer progression in patients with high lymph node ratios. Oncotarget 8, 74567-74581 https://doi.org/10.18632/oncotarget.20209

Jalali S, Bhartiya D, Lalwani MK, Sivasubbu S, Scaria V (2013): Systematic transcriptome wide analysis of lncRNA-miRNA interactions. PLoS ONE 8, e53823 https://doi.org/10.1371/journal.pone.0053823

Kobayashi H (2014): Imprinting genes associated with endometriosis. EXCLI J. 13, 252-264

Li B, Shi C, Li B, Zhao JM, Wang L (2018a): The effects of Curcumin on HCT-116 cells proliferation and apoptosis via the miR-491/ PEG10 pathway. J. Cell. Biochem. 119, 3091-3098 https://doi.org/10.1002/jcb.26449
Li J, Wu H, Li W, Yin L, Guo S, Xu X, Ouyang Y, Zhao Z, Liu S, Tian Y, Tian Z, Ju J, Ni B, Wang H (2016a): Downregulated miR-506 expression facilitates pancreatic cancer progression and chemoresistance via SPHK1/Akt/NF-kappaB signaling. Oncogene 35, 5501-5514 https://doi.org/10.1038/onc.2016.90

Li J, Zhang M, An G, Ma Q (2016b): LncRNA TUG1 acts as a tumor suppressor in human glioma by promoting cell apoptosis. Exp. Biol. Med. (Maywood) 241, 644-649 https://doi.org/10.1177/1535370215622708

Li J, Zhou L (2018b): Overexpression of lncRNA DANCR positively affects progression of glioma via activating Wnt/beta-catenin signaling. Biomed. Pharmacother. 102, 602-607 https://doi.org/10.1016/j.biopha.2018.03.116

Li X, Xiao R, Tembo K, Hao L, Xiong M, Pan S, Yang X, Yuan W, Xiong J, Zhang Q (2016c): PEG10 promotes human breast cancer cell proliferation, migration and invasion. Int. J. Oncol. 48, 1933-1942 https://doi.org/10.3892/ijo.2016.3406

Liu Z, Jiang Z, Huang J, Huang S, Li Y, Yu S, Yu S, Liu X (2014): miR-7 inhibits glioblastoma growth by simultaneously interfering with the PI3K/ATK and Raf/MEK/ERK pathways. Int. J. Oncol. 44, 1571-1580 https://doi.org/10.3892/ijo.2014.2322

Luo Y, Sun R, Zhang J, Sun T, Liu X, Yang B (2015): miR-506 inhibits the proliferation and invasion by targeting IGF2BP1 in glioblastoma. Am. J. Transl. Res. 7, 2007-2014

Malla RR, Gopinath S, Gondi CS, Alapati K, Dinh DH, Gujrati M, Rao JS (2011): Cathepsin B and uPAR knockdown inhibits tumor-induced angiogenesis by modulating VEGF expression in glioma. Cancer Gene Ther. 18, 419-434

https://doi.org/10.1038/cgt.2011.9

McCubrey JA, Steelman LS, Chappell WH, Abrams SL, Wong EW, Chang F, Lehmann B, Terrian DM, Milella M, Tafuri A, et al. (2007): Roles of the Raf/MEK/ERK pathway in cell growth, malignant transformation and drug resistance. Biochim. Biophys. Acta 1773, 1263-1284 https://doi.org/10.1016/j.bbamcr.2006.10.001

Miller JJ, Shih HA, Andronesi OC, Cahill DP (2017): Isocitrate dehydrogenase-mutant glioma: Evolving clinical and therapeutic implications. Cancer 123, 4535-4546 https://doi.org/10.1002/cncr.31039

Necsulea A, Soumillon M, Warnefors M, Liechti A, Daish T, Zeller U, Baker JC, Grutzner F, Kaessmann H (2014): The evolution of lncRNA repertoires and expression patterns in tetrapods. Nature 505, 635-640 https://doi.org/10.1038/nature12943

Okabe H, Satoh S, Furukawa Y, Kato T, Hasegawa S, Nakajima Y, Yamaoka Y, Nakamura Y (2003): Involvement of PEG10 in human hepatocellular carcinogenesis through interaction with SIAH1. Cancer Res. 63, 3043-3048

Ono R, Kobayashi S, Wagatsuma H, Aisaka K, Kohda T, KanekoIshino T, Ishino F (2001): A retrotransposon-derived gene, PEG10, is a novel imprinted gene located on human chromosome 7q21. Genomics 73, 232-237 https://doi.org/10.1006/geno.2001.6494

Paraskevopoulou MD, Hatzigeorgiou AG (2016): Analyzing MiRNA-LncRNA Interactions. Methods Mol. Biol. 1402, 271-286 
https://doi.org/10.1007/978-1-4939-3378-5_21

Peng T, Zhou L, Zuo L, Luan Y (2016): MiR-506 functions as a tumor suppressor in glioma by targeting STAT3. Oncol. Rep. 35, 1057-1064 https://doi.org/10.3892/or.2015.4406

Peng YP, Zhu Y, Yin LD, Zhang JJ, Wei JS, Liu X, Liu XC, Gao WT, Jiang KR, Miao Y (2017): PEG10 overexpression induced by E2F-1 promotes cell proliferation, migration, and invasion in pancreatic cancer. J. Exp. Clin. Cancer Res. 36, 30 https://doi.org/10.1186/s13046-017-0500-x

Shyu YC, Lee TL, Lu MJ, Chen JR, Chien RN, Chen HY, Lin JF, Tsou AP, Chen YH, Hsieh CW, Huang TS (2016): miR-122-mediated translational repression of PEG10 and its suppression in human hepatocellular carcinoma. J. Transl. Med. 14, 200 https://doi.org/10.1186/s12967-016-0956-z

Stewart LA (2002): Chemotherapy in adult high-grade glioma: a systematic review and meta-analysis of individual patient data from 12 randomised trials. Lancet (London, England) 359, 1011-1018 https://doi.org/10.1016/S0140-6736(02)08091-1

Tang WW, Wu Q, Li SQ, Tong YS, Liu ZH, Yang TX, Xu Y, Cao XF (2015): Implication of lncRNAs in pathogenesis of esophageal cancer. Onco Targets Ther. 8, 3219-3226 https://doi.org/10.2147/OTT.S87856

van der Zee M, Sacchetti A, Cansoy M, Joosten R, Teeuwssen M, Heijmans-Antonissen C, Ewing-Graham PC, Burger CW, Blok LJ, Fodde R (2015): IL6/JAK1/STAT3 Signaling blockade in endometrial cancer affects the ALDHhi/CD126+ stem-like component and reduces tumor burden. Cancer Res. 75, 3608-3622 https://doi.org/10.1158/0008-5472.CAN-14-2498

Wang J, Chu XQ, Zhang D, Kong DF (2018): Knockdown of long non-coding RNA PEG10 inhibits growth, migration and invasion of gastric carcinoma cells via up-regulating miR-3200. Neoplasma 65, 769-778 https://doi.org/10.4149/neo_2018_171204N794

Wen W, Liang W, Wu J, Kowolik CM, Buettner R, Scuto A, Hsieh MY, Hong H, Brown CE, Forman SJ, et al. (2014): Targeting JAK1/STAT3 signaling suppresses tumor progression and me- tastasis in a peritoneal model of human ovarian cancer. Mol. Cancer Ther. 13, 3037-3048

https://doi.org/10.1158/1535-7163.MCT-14-0077

Winter SF, Loebel F, Dietrich J (2017): Role of ketogenic metabolic therapy in malignant glioma: A systematic review. Crit. Rev. Oncol. Hematol. 112, 41-58 https://doi.org/10.1016/j.critrevonc.2017.02.016

Xing D, Liang JQ, Li Y, Lu J, Jia HB, Xu LY, Ma XL (2014): Identification of long noncoding RNA associated with osteoarthritis in humans. Orthop. Surg. 6, 288-293 https://doi.org/10.1111/os.12147

Xu MX, Zhao L, Deng C, Yang L, Wang Y, Guo T, Li L, Lin J, Zhang L (2013): Curcumin suppresses proliferation and induces apoptosis of human hepatocellular carcinoma cells via the wnt signaling pathway. Int. J. Oncol. 43, 1951-1959 https://doi.org/10.3892/ijo.2013.2107

Yao J, Zhou B, Zhang J, Geng P, Liu K, Zhu Y, Zhu W (2014): A new tumor suppressor LncRNA ADAMTS9-AS2 is regulated by DNMT1 and inhibits migration of glioma cells. Tumour Biol. 35, 7935-7944 https://doi.org/10.1007/s13277-014-1949-2

Zang W, Wang T, Huang J, Li M, Wang Y, Du Y, Chen X, Zhao G (2015): Long noncoding RNA PEG10 regulates proliferation and invasion of esophageal cancer cells. Cancer Gene Ther. 22, 138-144 https://doi.org/10.1038/cgt.2014.77

Zhang X, Sun S, Pu JK, Tsang AC, Lee D, Man VO, Lui WM, Wong ST, Leung GK (2012): Long non-coding RNA expression profiles predict clinical phenotypes in glioma. Neurobiol. Dis. 48, 1-8 https://doi.org/10.1016/j.nbd.2012.06.004

Zhang Z, Ma J, Luan G, Kang L, Su Y, He Y, Luan F (2015): MiR-506 suppresses tumor proliferation and invasion by targeting FOXQ1 in nasopharyngeal carcinoma. PLoS ONE 10, $\mathrm{e} 0122851$ https://doi.org/10.1371/journal.pone.0122851

Received: December 19, 2018

Final version accepted: May 10, 2019

First published online: June 26, 2019 